\title{
LARGE-SCALE STOCHASTIC HEREDITARY SYSTEMS UNDER MARKOVIAN STRUCTURAL PERTURBATIONS. PART II. QUALITATIVE ANALYSIS OF ISOLATED SUBSYSTEMS
}

\author{
G. S. LADDE \\ Received 14 January 2005; Revised 26 October 2005; Accepted 26 October 2005
}

In this part of the work, the convergence and stability analysis of isolated subsystems of stochastic hereditary systems under random structural perturbations is investigated. The variational comparison theorems developed in Part I are used to achieve this goal. Under algebraic conditions on the rate coefficients, time-delay, and an intensity matrix associated with the Markov chain, convergence and stability results are obtained. Furthermore, it is shown that these properties are affected by hereditary and random structural perturbations effects. It is further shown that the mathematical conditions are algebraically simple and are robust to the parametric changes. This investigation provides a basis for drawing the conclusions about the overall large-scale system.

Copyright (c) 2006 G. S. Ladde. This is an open access article distributed under the Creative Commons Attribution License, which permits unrestricted use, distribution, and reproduction in any medium, provided the original work is properly cited.

\section{Introduction}

The qualitative analysis plays a very important role in the study of a variety of dynamic processes in the engineering, medical, social, and physical sciences. In particular, the stability and convergence analysis is one of the most useful study for the benefit of mankind. The real world problems are highly nonlinear, nonstationary, and complex. Historically $[12,14,17,19-23]$, the Lyapunov's second method has played an important role in the study of qualititative properties of dynamic systems governed by systems of differential equations. In this part of the work, by employing the comparison results developed in $[11$, Part I], the stability and convergence results are established for isolated subsystems of a large-scale stochastic hereditary system. This provides a basis for the study of hierarchic systems. In Section 2, for easy reference, basic concepts of convergence and stability are presented. In Section 3, sufficient conditions in the context of the comparison theorems that insure convergence and stability of isolated stochastic hereditary subsystem are presented. Finally, to illustrate the scope and significance of the study, a simple example is 
presented in Section 4. The presented work extend and generalize the earlier work [1, 3$6,8,9,12-15,17-23]$ in a systematic and unified way.

\section{Qualitative properties}

In this section, we utilize the comparison theorems developed in [11, Part I] to investigate certain qualitative properties of isolated/decoupled subsystems [11]:

$$
d x^{i}=\mathbf{a}^{i}\left(t, x_{t}^{i}, \eta(t)\right) \cdot d \boldsymbol{\xi}(t), \quad x_{t_{0}}^{i}=\varphi_{0}^{i}
$$

where, $\mathbf{a}^{i}=\left(a_{0}^{i T}, a_{1}^{i T}, \ldots, a_{j}^{i T}, \ldots, a_{m}^{i T}\right)^{T}$ and $a_{j}^{i} \in C\left[J \times C^{n_{i}} \times R, R^{n_{i}}\right]$ for $j \in I(0, m)$; for each $i \in I(1, p)$. In particular, sufficient conditions are given to insure the convergence and stability of (DHS) depending on different modes of convergence in the probabilistic analysis $[2,14-17,21-23]$. The presented sufficient conditions relate the rate coefficients, the magnitude of the past-history, and the elements of the intensity matrix associated with the Markov chain in a systematic and natural way.

For easy reference, we present several types of convergence and stability concepts depending on different modes of convergence $[2,14,17,21,23]$ in probabilistic analysis. Without loss of generality, it is assumed that the convergence is to zero, and the stability is with respect to the trivial solution process of (DHS).

Definition $2.1[14,16]$. A solution process $x^{i}(t)=x\left(t_{0}, \varphi_{0}^{i}\right)(t)$ of stochastic system (DHS) is said to be as follows:

(i) convergent in the $\gamma$ th mean to zero if, for every $\epsilon>0, \delta>0$, and $t_{0} \in R_{+}$, there exists $T=T\left(\epsilon, \delta, t_{0}\right)>0$ such that

$$
\left(E\left[\left\|\varphi_{0}^{i}\right\|_{0}^{\gamma}\right]\right)^{1 / \gamma} \leq \delta
$$

implies

$$
\left(E\left[\left\|x^{i}\left(t_{0}, \varphi_{0}^{i}\right)(t)\right\|^{\gamma}\right]\right)^{1 / \gamma}<\epsilon \quad \text { for } t \geq T+t_{0}
$$

(ii) converge in probability to zero if, for every $\epsilon>0, t_{0} \in R$, and $0<\zeta<1$, there exists a $\delta>0$ and a $T=T\left(\epsilon, \delta, t_{0}\right)>0$ such that

$$
P\left\{\omega:\left\|\varphi_{0}^{i}\right\|_{0}>\delta\right\}<\zeta
$$

implies

$$
P\left\{\omega:\left\|x^{i}\left(t_{0}, \varphi_{0}^{i}\right)(t)\right\| \geq \epsilon\right\}<\zeta \quad \text { for } t \geq T+t
$$

(iii) almost sure convergent to zero if

$$
P\left\{\omega: \lim _{k \rightarrow \infty}\left\|x^{i}\left(t_{0}, \varphi_{0}^{i}\right)(t)\right\|=0\right\}=1 .
$$


Definition $2.2[3,5,14]$. The trivial solution process $x \equiv 0$ of stochastic hereditary system (DHS) is said to be as follows:

(i) stable in the $\gamma$ th mean if, for each $\epsilon>0, t_{0} \in R$, and $\gamma \geq 1$, there exists a positive function $\delta=\delta\left(t_{0}, \epsilon\right)$ such that the inequality

$$
\left(E\left[\left\|\varphi_{0}^{i}\right\|_{0}^{\gamma}\right]\right)^{1 / \gamma} \leq \delta
$$

implies

$$
\left(E\left[\left\|x^{i}\left(t_{0}, \varphi_{0}^{i}\right)(t)\right\|^{\gamma}\right]\right)^{1 / \gamma}<\epsilon, \quad \text { for } t \geq t_{0},
$$

(ii) asymptotically stable in the $\gamma$ th mean if it is stable in the $\gamma$ th mean and if, for any $\epsilon>0$ and $t_{0} \in R_{+}$, there exists a positive function $\delta_{1}=\delta_{1}\left(t_{0}\right)$ and a $T=T\left(\epsilon, \delta, t_{0}\right)>$ 0 such that the inequality

$$
\left(E\left[\left\|\varphi_{0}^{i}\right\|_{0}^{\gamma}\right]\right)^{1 / \gamma} \leq \delta_{1}
$$

implies

$$
\left(E\left[\left\|x^{i}\left(t_{0}, \varphi_{0}^{i}\right)(t)\right\|^{\gamma}\right]\right)^{1 / \gamma}<\epsilon, \quad \text { for } t \geq T+t_{0},
$$

(iii) stable in probability if, for each $\epsilon>0,1>\zeta>0, \delta>0$, and $t_{0} \in R_{+}$, there exists a positive function $\delta=\delta\left(t_{0}, \epsilon, \zeta\right)$ such that the inequality

$$
P\left\{\omega:\left\|\varphi_{0}^{i}\right\|_{0}>\delta\right\}<\zeta
$$

implies

$$
P\{\omega:\|x(k, \omega)\| \geq \epsilon\}<\zeta, \quad \text { for } t \geq t_{0},
$$

(iv) asymptotically stable in probability if it is stable in probability and if, for any $\epsilon>0$, $1>\zeta>0, \zeta>0$, and $t_{0} \in R_{+}$, there exists a positive function $\delta_{1}=\delta_{1}\left(t_{0}\right)$ and a $T=T\left(\epsilon, \delta, t_{0}\right)>0$ such that

$$
P\left\{\omega:\left\|\varphi_{0}^{i}\right\|_{0}>\delta_{1}\right\}<\zeta
$$

implies

$$
P\left\{\omega:\left\|x^{i}\left(t_{0}, \varphi_{0}^{i}\right)(t)\right\| \geq \epsilon\right\}<\zeta, \quad \text { for } t \geq T+t_{0},
$$

(v) almost surely stable if, for each $\epsilon>0$ and $t_{0} \in R_{0}$, there exists a positive function $\delta=\delta\left(t_{0}, \epsilon\right)$ such that the inequality

$$
\left\|\varphi_{0}^{i}\right\|_{0} \leq \delta
$$

with probability one implies

$$
\left\|x^{i}\left(t_{0}, \varphi_{0}^{i}\right)(t)\right\|<\epsilon, \quad \text { for } t \geq t_{0}
$$

with probability one, 
(vi) almost surely asymptotically stable if it is almost surely stable with probability one and if, for any $\epsilon>0$ and $t_{0} \in R_{+}$, there exists a positive function $\delta_{1}=\delta_{1}\left(t_{0}\right)$ and a $T=T\left(\epsilon, \delta, t_{0}\right)>0$ such that the inequality

$$
\left\|\varphi_{0}^{i}\right\|_{0} \leq \delta_{1}
$$

with probability one implies

$$
\left\|x^{i}\left(t_{0}, \varphi_{0}^{i}\right)(t)\right\|<\epsilon, \quad \text { for } t \geq T+t_{0}
$$

with probability one.

In order to prove the convergence and stability results for (DHS), we need to define the convergence and stability concepts corresponding to Definitions 2.1 and 2.2 for the solution process $r^{i}\left(t_{0}, \sigma_{0}^{i}\right)(t)$ of comparison system (BCS)/(LCS) [11]:

$$
\frac{d}{d t} u^{i}=w^{i}\left(t, u^{i}, u_{t}^{i}\right), \quad u_{t_{0}}^{i}=\sigma_{0}^{i}
$$

existing for $t \geq t_{0}$ for $\left(t_{0}, \sigma_{0}^{i}\right) \in R_{+} \times\left(\mathscr{C}_{+}^{q_{i}}\right)^{q}$ and each $i \in I(1, p)$.

Again, in this definition, it is also assumed that the solution process of (BCS) convergence is to zero.

Definition 2.3. The maximal solution process $r^{i}\left(t_{0}, \sigma_{0}^{i}\right)(t)$ of (BCS) [11] through $\left(t_{0}, \sigma_{0}^{i}\right)$ is said to converge to zero if for every $\epsilon>0$ and $\delta>0$, and $t_{0} \in J$, there exists a positive number $T=T\left(\epsilon, \delta, t_{0}\right)>0$ such that

$$
\sum_{j=1}^{q} \sum_{l=1}^{q_{i}} E\left[\sigma_{0 l}^{i j}\right] \leq \delta
$$

implies for

$$
\sum_{j=1}^{q} \sum_{l=1}^{q_{i}} E\left[r_{l}^{i j}\left(t, t_{0}, \sigma_{0}^{i}\right)\right]<\epsilon
$$

for $t \geq T+t_{0}$.

Similarly, the stability concepts with regard to comparison system (BCS) and other stability concepts with regard to both (DHS) and (BCS) can be reformulated, analogously. For details, see $[5,14,17,22]$.

\section{Qualitative analysis of isolated subsystem}

Now, depending on different modes of convergence, we are ready to present convergence and stability results. The following result deals with $\gamma$ th mean convergence result. 
Theorem 3.1. Assume that hypotheses of [11, Theorem 3.1] are satisfied. Further assume that

(i) for each $t \in R_{+}, V^{i}(t, x, j)$ satisfies the inequality

$$
a\left(\left\|x^{i}\right\|^{\gamma}\right) \leq \sum_{j=1}^{q} \sum_{k=1}^{q_{i}} V_{k}^{i}(t, x, j) \leq b\left(\left\|x^{i}\right\|^{\gamma}\right),
$$

where $a \in \mathscr{V} \mathscr{K}$ and $b \in \mathscr{C} \mathcal{K}$,

(ii) the maximal solution process $r^{i}\left(t_{0}, \sigma_{0}^{i}\right)(t)$ of (BCS) converges to zero.

Then, the solution process $x^{i}\left(t_{0}, \varphi_{0}^{i}\right)(t)$ of (DHS) converges in the $\gamma$ th mean to the zero.

Proof. The proof of the result can be constructed by imitating the arguments used in the proofs of results in $[1,3,14,17]$. The details are left to the reader.

In the following, we provide the sufficient conditions to insure the almost sure convergence of a solution process of (DHS) to the zero vector.

Theorem 3.2. Assume that hypotheses of [11, Theorem 3.1] are satisfied. Further assume that

(i) the function $V^{i}\left(t, x^{i}, y\right)$ is such that $V^{i}(t, 0, y) \equiv 0$ is the unique minimum of $V^{i}\left(t, x^{i}\right.$, $y)$, where $0 \in R_{+}^{q_{i}}$ denotes the zero vector,

(ii) there exists a matrix function $\Upsilon^{i}(t)$ such that $\Upsilon^{i} \in \mathscr{C}\left[\mathscr{R}_{+},\left(R_{+}^{q_{i}}\right)^{q} \times\left(R_{+}^{q_{i}}\right)^{q}\right]$ and is a block diagonal matrix which is defined by

$$
\begin{aligned}
\Upsilon^{i}(t) & =\operatorname{diag}\left\{T^{i 1}(t), T^{i 2}(t), \ldots, T^{i j}(t), \ldots, T^{i q}(t)\right\}, \\
T^{i j}(t) & =\operatorname{diag}\left\{\kappa_{11}^{i j}(t), \kappa_{22}^{i j}(t), \ldots, \kappa_{q_{t} q_{t}}^{i j}(t)\right\}_{q_{t} \times q_{t}},
\end{aligned}
$$

where $\kappa_{k k}^{i j}(t)>0$ for $1 \leq i \leq p, 1 \leq k \leq q_{i}$, and for $u^{i}, v^{i} \in\left(R_{+}^{q_{i}}\right)^{q}, u^{i} \geq v$,

$$
w^{i}\left(t, u^{i}, u_{t}^{i}\right)-w^{i}\left(t, v^{i}, v_{t}^{i}\right) \geq-\Upsilon^{i}(t)\left(u^{i}-v^{i}\right)
$$

(iii)

$$
\int_{t_{0}}^{\infty}\left[w^{i}\left(t, E\left[r^{i}(t)\right], E\left[r_{t}^{i}\right)\right]+\Upsilon^{i}(t) E\left[r^{i}(t)\right]\right] d t<\infty
$$

where $r^{i}(t)=r^{i}\left(t_{0}, \sigma_{0}^{i}\right)(t)$ is the maximal solution $u^{\prime}=w^{i}\left(t, u^{i}, \sigma^{i}\right)$, through $\left(t_{0}, \sigma_{0}^{i}\right)$ and

$$
w^{i}(t, 0,0) \geq 0, \quad \text { for } t \geq t_{0}
$$

(iv) the mean of the maximal solution $E\left[r^{i}(t)\right]=E\left[r\left(t_{0}, \sigma_{0}^{i}\right)(t)\right]$ of $(B C S)$ through $\left(t_{0}, \sigma_{0}^{i}\right)$ converges to the zero vector as $t \rightarrow \infty$.

Then a solution process of (DHS) converges to the zero vector almost surely as $t \rightarrow \infty$. 
6 Large-scale stochastic systems. Part II. Isolated subsystems

Proof. For $t_{0} \leq s \leq t$, we define a function $Z: R_{+} \times\left(R_{+}^{q_{i}}\right)^{q} \times\left(C_{+}^{q_{i}}\right)^{q} \rightarrow R_{+}^{q_{i}}$ by

$$
\begin{aligned}
Z_{j}^{i}(s)= & T^{i j}(s) V^{i}\left(s, z^{i}\left(t, s, x^{i}\left(t_{0}, \varphi_{0}^{i}\right)(s)\right), \eta(s)\right) \\
& +g^{i j}\left(s, V^{i}\left(s, z^{i}\left(t, s, x^{i}\left(t_{0}, \varphi_{0}^{i}\right)(s)\right), \eta(s)\right), V_{s}^{i}\right) \\
& +\sum_{l \neq \eta(s)}^{q} \lambda_{\eta(s) l} V^{i}\left(s, z^{i}\left(t, s, x^{i}\left(t_{0}, \varphi_{0}^{i}\right)(s)\right), l\right) .
\end{aligned}
$$

From the assumption of the theorem $E\left[Z_{j}^{i}(s)\right]$ exists. Moreover, we note that $\int_{t_{0}}^{s} Z_{j}^{i}(v) d v$ exists and nondecreasing in $s$. Under the assumptions on $g^{i j}$ and $r^{i j}\left(t_{0}, \sigma_{0}^{i}\right)(s)$, the following inequalities

$$
\begin{aligned}
\int_{t_{0}}^{s} E\left[Z_{j}^{i}(v)\right] d v \leq \int_{t_{0}}^{s}[ & T^{i j}(v) E\left[r^{i j}\left(t_{0}, \sigma_{0}^{i}\right)(v)\right]+g^{i j}\left(v, E\left[r^{i j}\left(t_{0}, \sigma_{0}^{i}\right)(s)\right]\right), E\left[r_{v}^{i j}\right] \\
& \left.+\sum_{l \neq j}^{q} \lambda_{j l} E\left[r^{i l}\left(t_{0}, \sigma_{0}^{i}\right)(s)\right]\right] d s, \\
\int_{t_{0}}^{\infty} E\left[Z_{j}^{i}(s) \geq\right] d s \leq \int_{t_{0}}^{\infty}[ & T^{i j}(s) E\left[r^{i j}\left(t_{0}, \sigma_{0}^{i}\right)(s)\right]+g^{i j}\left(s, E\left[r^{i j}\left(t_{0}, \sigma_{0}^{i}\right)(s)\right]\right), E\left[r_{s}^{i j}\right] \\
& \left.+\sum_{l \neq j}^{q} \lambda_{j l} E\left[r^{i l}\left(t_{0}, \sigma_{0}^{i}\right)(s)\right]\right] d s
\end{aligned}
$$

are valid. From (3.8) and assumption (iii), we conclude that $\int_{t_{0}}^{\infty} E\left[Z_{j}^{i}(s)\right] d s<\infty$. We set $\int_{s}^{\infty}\left[Z_{j}^{i}(v)\right] d v=\int_{t_{0}}^{\infty}\left[Z_{j}^{i}(v)\right] d v-\int_{t_{0}}^{s} Z_{j}^{i}(v) d v$. For each $i \in I(1, p)$, we now define

$$
W^{i}\left(s, z^{i}\left(t, s, \varphi^{i}(0)\right), \eta(s)\right)=V^{i}\left(s, z^{i}\left(t, s, \varphi^{i}(0)\right), \eta(s)\right)+\int_{s}^{\infty} Z_{j}^{i}(v) d v .
$$

Applying the operators defined in [11, Theorem 3.1, in particular, (3.1), (3.2), and (3.3)] to $W^{i}$ in (3.9) along a solution process $x^{i}\left(t_{0}, \varphi_{0}^{i}\right)(s)$ of (DHS) and utilizing hypotheses of [11, Theorem 3.1, in particular, (3.5), (3.7), (3.8)] and the definition of $Z_{j}^{i}$ in (3.6), we obtain

$$
\begin{aligned}
D_{(\mathrm{DHS})}^{+} & W^{i}\left(s, \varphi^{i}, z^{i}\left(t, s, \varphi^{i}(0)\right), \eta(s)\right) \\
\leq & L_{i}^{D} W^{i}\left(s, \varphi^{i}, z^{i}\left(t, s, \varphi^{i}(0)\right), j\right)+D_{\eta}^{+} W^{i}\left(s, z^{i}\left(t, s, \varphi^{i}(0)\right), j\right) \\
\leq & L_{i}^{D} V^{i}\left(s, \varphi^{i}, z^{i}\left(t, s, \varphi^{i}(0)\right), j\right)+D_{\eta}^{+} V^{i}\left(s, z^{i}\left(t, s, \varphi^{i}(0)\right), j\right)-Z_{j}^{i}(s) \\
\leq & g^{i j}\left(s, V^{i}\left(s, z^{i}\left(t, s, \varphi^{i}(0)\right), \eta(s)\right), V_{s}^{i}\right)-Z_{j}^{i}(s) \\
& +D_{\eta}^{+} V^{i}\left(s, z^{i}\left(t, s, \varphi^{i}(0)\right), j\right) \leq-\left(\pi_{j j} I+T^{i j}(s)\right) V^{i}\left(s, z^{i}\left(t, s, \varphi^{i}(0)\right), \eta(s)\right),
\end{aligned}
$$

where $\varphi^{i}(0)=x^{i}\left(t_{0}, \varphi_{0}^{i}\right)(s)$ for $t_{0} \leq s \leq t$. From the definitions of $\pi_{j j}$ and $T^{i j}$, the nature 
of $V^{i}$, and (3.10), it is clear that

$$
D_{(\mathrm{DHS})}^{+} W^{i}\left(s, \varphi^{i}, z^{i}\left(t, s, x^{i}\left(t_{0}, \varphi_{0}^{i}\right)(s)\right), \eta(s)\right) \leq 0 .
$$

Also, from the assumption of the existence of $E\left[V^{i}\left(t_{0}, z^{i}\left(t, t_{0}, \varphi_{0}^{i}(0)\right), \eta\left(t_{0}\right)\right)\right],(3.4),(3.8)$, and (3.9), we obtain

$$
\begin{aligned}
E\left[W^{i}\left(t_{0}, z^{i}\left(t, t_{0}, \varphi_{0}^{i}(0)\right), \eta\left(t_{0}\right)\right)\right] \\
=E\left[V^{i}\left(t_{0}, z^{i}\left(t, t_{0}, \varphi_{0}^{i}(0)\right), \eta\left(t_{0}\right)\right)\right]+\int_{t_{0}}^{\infty} E\left[Z_{j}^{i}(s)\right] d s \\
\leq E\left[V^{i}\left(t_{0}, z^{i}\left(t, t_{0}, \varphi_{0}^{i}(0)\right), \eta\left(t_{0}\right)\right)\right] \\
\quad+\int_{t_{0}}^{\infty}\left[w^{i j}\left(t, E\left[r^{i}(t)\right], E\left[r_{t}^{i}\right]\right)+T^{i j}(t) E\left[r^{i j}(t)\right]\right] d t<\infty .
\end{aligned}
$$

The nonnegativity of $W^{i},(3.11),(3.12)$, and an application of well-known result $[2,21]$ gives us that $W^{i}\left(t, x^{i}\left(t_{0}, \varphi_{0}^{i}\right)(t), \eta(t)\right)$ is non-negative supermartingale. Therefore, from the property of nonnegativity of the supermartingale, $W^{i}\left(t, x^{i}\left(t_{0}, \varphi_{0}^{i}\right)(t), \eta(t)\right)$ converges almost surely as $t \rightarrow \infty$. From (3.8) and (3.9), it is clear that $V^{i}\left(t, x^{i}\left(t_{0}, \varphi_{0}^{i}\right)(t), \eta(t)\right)$ converges almost surely as $t \rightarrow \infty$. In order to conclude the proof of the theorem, we first need to show that $V^{i}\left(t, x^{i}\left(t_{0}, \varphi_{0}^{i}\right)(t), \eta(t)\right) \rightarrow 0$ as $t \rightarrow \infty$. For this purpose, we note from [11, Theorem 3.1] that for $i \in I\left(1, q_{i}\right)$ and $j \in I(1, q)$,

$$
E\left[V^{i}\left(t, x^{i}(t), j\right)\right] \leq E\left[r^{i j}\left(t_{0}, \sigma_{0}^{i}\right)(t)\right], \quad t \geq t_{0}
$$

Assumption (iv) of the theorem provides us that $E\left[r^{i}\left(t_{0}, \sigma_{0}^{i}\right)(t)\right] \rightarrow 0$ as $t \rightarrow \infty$. From this and (3.13) and the application of Fatou's lemma $[2,14,21]$, we have

$$
\lim _{t \rightarrow \infty} E\left[V^{i}\left(t, x^{i}(t), j\right)\right] \leq 0
$$

From assumption (i), we have

$$
\lim _{t \rightarrow \infty} E\left[V^{i}\left(t, x^{i}(t), j\right)\right] \geq 0
$$

and therefore from (3.14), we obtain $\lim _{t \rightarrow \infty} E\left[V^{i}\left(t, x^{i}(t), j\right)\right]=0$. This together with the properties of expectation establishes $\lim _{t \rightarrow \infty}\left[V^{i}\left(t, x^{i}(t), j\right)\right]=0$. From this, assumption (i), and continuity of $V^{i}$, the almost sure convergence of the solution process of (DHS) to zero follows, immediately. This completes the proof.

Now, we present sufficient conditions that assure the $\gamma$ th mean and almost sure stability properties of the trivial solution process of (DHS). 
8 Large-scale stochastic systems. Part II. Isolated subsystems

Theorem 3.3. Assume that hypotheses of [11, Theorem 3.1] are satisfied. Further assume that

(i) for each $t \in R_{+}, V^{i}(t, x, j)$ satisfies the inequality

$$
a\left(\left\|x^{i}\right\|^{\gamma}\right) \leq \sum_{j=1}^{q} \sum_{k=1}^{q_{i}} V_{k}^{i}(t, x, j) \leq b\left(\left\|x^{i}\right\|^{\gamma}\right),
$$

where $a \in \mathscr{V} \mathscr{K}$ and $b \in \mathscr{C K}$,

(ii) $a^{i}(t, 0, \eta(t)) \equiv 0$ and $w(t, 0,0) \equiv 0$.

Then,

(a) the stability of trivial solution of $r^{i}(t) \equiv 0$ of $(B C S)$ implies the $\gamma$ th mean stability of the trivial solution process $x^{i}(t) \equiv 0$ of (DHS),

(b) the asymptotic stability of trivial solution of $r^{i}(t) \equiv 0$ of (BCS) implies the $\gamma$ th mean asymptotic stability of the trivial solution process $x^{i}(t) \equiv 0$ of (DHS).

Proof. The proof of the theorem can be formulated by imitating the proofs of theorems in $[3,5,6,10]$. However, the detailed proofs will appear elsewhere.

Theorem 3.4. Assume that hypotheses of [11, Theorem 3.1] are satisfied. Further assume that

(i) for each $t \in R_{+}, V^{i}(t, x, j)$ satisfies the inequality

$$
a\left(\left\|x^{i}\right\|^{\gamma}\right) \leq \sum_{j=1}^{q} \sum_{k=1}^{q_{i}} V_{k}^{i}(t, x, j) \leq b\left(\left\|x^{i}\right\|^{\gamma}\right),
$$

where $a \in \mathscr{V} \mathscr{K}$ and $b \in \mathscr{C} \mathscr{K}$,

(ii) there exists a matrix function $\Upsilon^{i}(t)$ such that $\Upsilon^{i} \in \mathscr{C}\left[\mathscr{R}_{+},\left(R_{+}^{q_{i}}\right)^{q} \times\left(R_{+}^{q_{i}}\right)^{q}\right]$ and is a block diagonal matrix which is defined by

$$
\begin{aligned}
& \Upsilon^{i}(t)=\operatorname{diag}\left\{T^{i 1}(t), T^{i 2}(t), \ldots, T^{i j}(t), \ldots, T^{i q}(t)\right\}, \\
& T^{i j}(t)=\operatorname{diag}\left\{\kappa_{11}^{i j}(t), \kappa_{22}^{i j}(t), \ldots, \kappa_{q_{t} q_{t}}^{i j}(t)\right\}_{q_{t} \times q_{t}},
\end{aligned}
$$

where $\kappa_{k k}^{i j}(t)>0$ for $1 \leq i \leq p, 1 \leq k \leq q_{i}$, and for $u^{i}, v^{i} \in\left(R_{+}^{q_{t}}\right)^{q}, u^{i} \geq v$,

$$
w^{i}\left(t, u^{i}, u_{t}^{i}\right)-w^{i}\left(t, v^{i}, v_{t}^{i}\right) \geq-\Upsilon^{i}(t)\left(u^{i}-v^{i}\right),
$$

(iii)

$$
\int_{t_{0}}^{\infty}\left[w^{i}\left(t, E\left[r^{i}(t)\right], E\left[r_{t}^{i}\right]\right)+\Upsilon^{i}(t) E\left[r^{i}(t)\right]\right] d t<\infty
$$

where $r^{i}(t)=r^{i}\left(t_{0}, \sigma_{0}^{i}\right)(t)$ is the maximal solution of $u^{\prime}=w^{i}\left(t, u^{i}, \sigma^{i}\right)$ through $\left(t_{0}, \sigma_{0}^{i}\right)$, (iv) $\mathbf{a}^{i}(t, 0, \eta(t)) \equiv 0$ and $w(t, 0,0) \equiv 0$.

Then,

(a) the stability of trivial solution of $r^{i}(t) \equiv 0$ of (BCS) implies the almost sure stability of the trivial solution process $x^{i}(t) \equiv 0$ of (DHS), 
(b) the asymptotic stability of trivial solution of $r^{i}(t) \equiv 0$ of (BCS) implies the $\gamma$ th mean asymptotic stability of the trivial solution process $x^{i}(t) \equiv 0$ of $(D H S)$.

Proof. The proof of the theorem can be constructed by employing the proof of Theorem 3.2. The details are left to the reader.

The following corollary illustrates the scope of Theorems 3.1, 3.2, 3.3, and 3.4.

Corollary 3.5. Assume that hypotheses of [11, Corollary 3.1] are satisfied. Further assume that

(i) the assumption (i) of Theorem 3.1 is satisfied;

(ii) $\mathbf{a}^{i}\left(t, x_{t}^{i}, \eta(t)\right) \equiv 0$,

(iii) the elements of matrices $\bar{A}^{i}(t), \bar{B}^{i}(t)$, and past-history $\tau$ satisfy the following inequalities:

$$
\begin{gathered}
\pi_{j j}-\alpha^{i j}(t)-\tau \beta^{i j}(t)>0, \quad j \in I(1, q), \\
\pi_{j j}-\alpha^{i j}(t)-\tau \beta^{i j}(t)-d_{j}^{-1} \sum_{l \neq j}^{q} d_{l} \pi_{l j}>\alpha(t)>0
\end{gathered}
$$

for some $d_{j}>0$ for every $j \in I(1, q)$. Then,

(a) $\alpha \in L^{1}\left[R_{+}, R_{+}\right]$implies the $\gamma$ th mean stability of the trivial solution process of (DHS),

(b) $\liminf _{\left(t-t_{0}\right) \rightarrow \infty}\left[\left(1 /\left(t-t_{0}\right)\right)\left(\int_{t_{0}}^{t} \alpha(\theta) d \theta\right)\right] \geq \bar{\alpha}>0$ implies the $\gamma$ th mean asymptotic stability of the trivial solution process of (DHS).

Proof. To conclude the validity of the conclusions of the corollary, it is enough to prove that

(1) assumptions of Theorem 3.3 are satisfied,

(2) $\alpha \in L^{1}\left[R_{+}, R_{+}\right]$implies the stability of the trivial solution process of (LCS) [11];

$$
\frac{d}{d t} u^{i}=-\bar{A}^{i}(t) u^{i}+L^{i}\left(u_{t}^{i}\right), \quad u_{t_{0}}^{i}=\sigma_{0}^{i}
$$

(3) $\liminf _{\left(t-t_{0}\right) \rightarrow \infty}\left[\left(1 /\left(t-t_{0}\right)\right)\left(\int_{t_{0}}^{t} \alpha(\theta) d \theta\right)\right] \geq \bar{\alpha}>0$ implies asymptotic stability of the trivial solution process of (LCS).

These statements together with the assumptions of corollary would fulfill all the hypotheses of Theorem 3.3. Hence, by the application of Theorem 3.3, the conclusions of the corollary follow, immediately. In fact, we first note that $w\left(t, u, u_{t}\right)=-\bar{A}^{i} u+L^{i}\left(u_{t}\right)$ satisfies the relation $w(t, 0,0) \equiv 0$. To prove the remaining statements (2) and (3), we use a scalar Lyapunov function for (LCS),

$$
v(t, u)=\sum_{k=1}^{s} d_{k} u_{k}
$$

ordinary differential inequality, and comparison theorem in the context of minimal class 
of functions defined by the following:

$$
\Omega_{A}=\left\{\varphi \in \mathscr{C}^{q}: \sup _{\theta \in[-\tau, 0]} A(t+\theta) v(t+\theta, \varphi(\theta)) \leq A(t) v(t, \varphi(0))\right\}
$$

where a real-valued continuous function $A$ is determined by

$$
G(\lambda)=\lambda+\sup _{j \in I(1, q)}\left\{-\pi_{j j}+\alpha^{i j}(t)+d_{j}^{-1} \sum_{l \neq k}^{q} d_{l} \pi_{l j}\right\}+\sup _{j \in I(1, q)}\left\{\tau \beta^{i j}(t) \exp [\tau \lambda]\right\} .
$$

From (3.21) and (3.24), we observe that $G(0)<0$, and $G(\lambda)$ is increasing on $(0, \infty)$. Therefore, we can find a positive number $\alpha$ such that $G(\alpha) \leq 0$. We can take $A(t)=\exp [\alpha t]$. The validity of the corollary follows by following the argument used in [7].

\section{Examples}

In the following, we present simple examples that illustrate the usefulness of the presented results of [11, Part I] as well as the results of this paper.

Example 4.1. We consider a very simple scalar version of (DHS) is as follows:

$$
d x=\left(-\frac{1}{2} x^{2}+a(\eta(t))\right) x d t+b(\eta(t)) x(t-\tau) d \xi_{1}(t), \quad x_{t_{0}}=\varphi_{0}
$$

where $a, b \in C[R, R], \eta$ is as defined before, $\tau>0$ is stochastic time-delay defined on the complete probability space $\left(\Omega, \mathscr{F}_{F}, P\right)$, and it is independent of the random processes $\xi_{1}$, $\eta$ and initial state process $\varphi_{0}$. Furthermore, $\tau \in[\nu, \mu]$ with probability one, where $\nu \geq 0$ and $\mu>0$. This assumption on $\tau$ characterizes the "reaction time-delay" in the hereditary system with past memory $\mu[4,9,10]$.

(i) Let $F$ be a distribution function of a random variable $\tau$ defined on a complete probability space $(\Omega, \mathscr{F}, P)$. Let us note that

$$
\int_{-\infty}^{\infty} d F(\theta)=\int_{\nu}^{\mu} d F(\theta)=1
$$

and it is obvious that (4.1) can be rewritten as

$$
d x=\left(-\frac{1}{2} x^{2}+a(\eta(t))\right) x d t+b(\eta(t)) \int_{\nu}^{\mu} x(t-\theta) d F(\theta) d \xi_{1}(t), \quad x_{t_{0}}=\varphi_{0}
$$

which implies that

$$
d x=\left(-\frac{1}{2} x^{2}+a(\eta(t))\right) x d t-b(\eta(t)) \int_{-\mu}^{-v} x(t+\theta) d F(-\theta) d \xi_{1}(t), \quad x_{t_{0}}=\varphi_{0} .
$$


(ii) Let $F$ in (i) be defined by

$$
F(\theta)= \begin{cases}0, & \text { if } \theta<v, \\ \frac{(\mu-\theta)}{(\mu-v)}, & \text { if } \nu \leq \theta \leq \mu, \\ 1, & \text { if } \mu<\theta .\end{cases}
$$

In this case, (4.4) reduces to

$$
d x=\left(-\frac{1}{2} x^{2}+a(\eta(t))\right) x d t+\frac{b(\eta(t))}{\mu-v} \int_{-\mu}^{-v} x(t+\theta) d \theta d \xi_{1}(t), \quad x_{t_{0}}=\varphi_{0}
$$

For this example, we consider the following scalar version of auxiliary differential equation (DHS) in [11] as:

$$
z^{\prime}=-\frac{1}{2} z^{3}, \quad z\left(t_{0}\right)=z_{0}
$$

We note that here $f(t, z)=-(1 / 2) z^{3}$ is twice differentiable with respect to $z$. The close form solution of (4.7) is

$$
z\left(t, t_{0}, z_{0}\right)=z_{0}\left[1+z_{0}^{2}\left(t-t_{0}\right)\right]^{-1 / 2}
$$

It is clear that the nontrivial solution of (4.7) is twice continuously differentiable with respect to $\left(t, t_{0}, z_{0}\right)$. Moreover, in this case

$$
\begin{aligned}
& \frac{\partial}{\partial x} z\left(t, t_{0}, z_{0}\right)=\Phi\left(t, t_{0}, z_{0}\right)=\left[1+z_{0}^{2}\left(t-t_{0}\right)\right]^{-3 / 2} \\
& \frac{\partial^{2}}{\partial x^{2}} z\left(t, t_{0}, z_{0}\right)=-3\left(t-t_{0}\right) z_{0}\left[1+z_{0}^{2}\left(t-t_{0}\right)\right]^{-5 / 2}
\end{aligned}
$$

We choose a Lyapunov-like function as $V(t, x, \eta(t))=(1 / 2) H(\eta(t))|x|^{2}$. In this case, the expressions in [11, in particular, (3.4)] reduce to the following: $M^{D}(s, \varphi(0), \varphi, \eta(s))=$ $a(\eta(s)) \varphi(0), \quad \Lambda^{D}(s, \varphi, \eta(s))=\left[b(\eta(t)) \int_{-\mu}^{-\nu} \varphi(0) d F(-\theta)\right]^{2}, \quad \Theta^{D}(t, s, \varphi, \eta(s))=[b(\eta(t)) \times$ $\left.\int_{-\mu}^{-v} \varphi(0) d F(-\theta)\right]^{2}\left[1+x^{2}(s)(t-s)\right]^{-3}$ for $t_{0} \leq s \leq t$, and

$$
\begin{aligned}
L^{D} V(s, \varphi, z(t, s, \varphi(0)), \eta(s)) \\
=\frac{1}{2} \operatorname{tr}\left(\frac{\partial^{2}}{\partial z^{2}} V(s, z(t, s, \varphi(0)), \eta(s)) \Theta^{D}(t, s, \varphi, \eta(s))\right)+\frac{\partial}{\partial z} V(s, z(t, s, \varphi(0)), \eta(s)) \\
\quad \times\left[\frac{1}{2} \operatorname{tr}\left(\frac{\partial^{2}}{\partial x \partial z} z(t, s, \varphi(0)) \Lambda^{D}(s, \varphi, \eta(s))\right)+\Phi(t, s, \varphi(0)) M^{D}(s, \varphi(0), \varphi, \eta(s))\right]
\end{aligned}
$$


12 Large-scale stochastic systems. Part II. Isolated subsystems

$$
\begin{aligned}
= & \frac{1}{2} H(\eta(s))\left[b(\eta(t)) \int_{-\mu}^{-\nu} \varphi(\theta) d F(-\theta)\right]^{2}\left[1+\varphi^{2}(0)(t-s)\right]^{-3} \\
& +H(\eta(s)) \varphi(0)\left[1+\varphi^{2}(0)(t-s)\right]^{-1 / 2} \\
& \times\left(a(\eta(s)) \varphi(0)\left[1+\varphi^{2}(0)(t-s)\right]^{-3 / 2}\right. \\
& \left.\quad-\frac{3}{2}(t-s) \varphi(0)\left[1+\varphi^{2}(0)(t-s)\right]^{-5 / 2}\left[b(\eta(t)) \int_{-\mu}^{-v} \varphi(\theta) d F(-\theta)\right]^{2}\right) \\
= & H(\eta(s))\left[1+\varphi^{2}(0)(t-s)\right]^{-2} a(\eta(s)) \varphi^{2}(0)+\left(1-3 \varphi^{2}(0)(t-s)\right) \\
& \times \frac{1}{2} H(\eta(s))\left[1+\varphi^{2}(0)(t-s)\right]^{-3}\left[b(\eta(t)) \int_{-\mu}^{-v} \varphi(\theta) d F(-\theta)\right]^{2} \\
\leq & 2 a(\eta(s)) \frac{1}{2} H(\eta(s)) z^{2}(t, s, \varphi(0)) \\
& +\frac{1}{2} H(\eta(s))\left[1+\varphi^{2}(0)(t-s)\right]^{-2}\left[b(\eta(t)) \int_{-\mu}^{-v} \varphi(\theta) d F(-\theta)\right]^{2} .
\end{aligned}
$$

From this and the Cauchy-Bunyakovski-Schwarz inequality and the nature of the solution of auxiliary differential equation (4.7), we have

$$
L^{D} V(s, \varphi, z(t, s, \varphi(0)), j) \leq 2 a(j) V(s, z(t, s, \varphi(0)), j)+b^{2}(j)\left\|V_{s}(j)\right\|_{0},
$$

where $\max _{\theta \in|-\mu,-\nu|} V(s+\theta, z(t, s+\theta, \varphi(\theta)), j)=\left\|V_{s}(j)\right\|_{0}$. The comparison system of functional differential equations is

$$
\frac{d}{d t} u=-\bar{A} u+\bar{B}\left\|u_{t}\right\|_{0}, \quad u_{t_{0}}=\sigma_{0}
$$

where

$$
\begin{gathered}
\bar{A}=\left(a_{j l}\right) q \times q, \quad \bar{B}=\operatorname{diag}\left(b^{2}(1), \ldots, b^{2}(j), \ldots, b^{2}(q)\right), \\
a_{j l}= \begin{cases}\pi_{j j}-2 a(j), & l=j, \\
-\pi_{j l}, & l \neq j .\end{cases}
\end{gathered}
$$

The trivial solution of (4.12) is asymptotically stable if

$$
\begin{aligned}
& \pi_{j j}-2 a(j)-\tau b^{2}(j)>0, \quad j \in I(1, q), \\
& \pi_{j j}-2 a(j)-\tau b^{2}(j)-d_{j}^{-1} \sum_{l \neq j}^{q} d_{l} \pi_{l j}>\alpha>0 .
\end{aligned}
$$


Hence, by the application of Theorem 3.3, the trivial solution process of (4.1) is asymptotically stable in the mean-square sense. Moreover, it can be proved that it is almost surely asymptotically stable.

Example 4.2. The asymptotic stability (exponential stability) in the mean of the trivial solution of (DHS) is assured if the coefficient of comparison system in [11, in particular, in (LCS)] satisfies the conditions

$$
\begin{aligned}
& \pi_{j j}-\alpha^{i j}(t)-\tau \beta^{i j}(t)>0, \quad j \in I(1, q), \\
& \pi_{j j}-a^{i j}(t)-\tau \beta^{i j}(t)-d_{j}^{-1} \sum_{l \neq j}^{q} d_{l} \pi_{l j}>\alpha>0
\end{aligned}
$$

for some $\alpha>0$ for each $i \in I(1, q)$.

Remark 4.3. It is clear that the sufficient conditions for the validity of qualitative properties of stochastic hereditary decoupled subsystems under Markovian structural perturbations are related with coefficients of intensity matrix $\Pi=\left(\pi_{l j}\right)_{q \times q}$, time-delay, and the estimates on the rate functions. Moreover, from (4.15) one can draw several implications about the effects of Markovian structural perturbations as well as the hereditary effects on systems.

\section{References}

[1] M. H. Chang, G. S. Ladde, and P. T. Liu, Stability of stochastic functional differential equations, Journal of Mathematical Physics 15 (1974), no. 9, 1474-1478.

[2] R. J. Elliott, Stochastic Calculus and Applications, Applications of Mathematics (New York), vol. 18, Springer, New York, 1982.

[3] G. S. Ladde, Differential inequalities and stochastic functional differential equations, Journal of Mathematical Physics 15 (1974), no. 6, 738-743.

[4] - Competitive processes. I. Stability of hereditary systems, Nonlinear Analysis. Theory, Methods \& Applications. Series A: Theory and Methods 1 (1977), no. 6, 607-631.

[5] _ Stability of general systems in biological, physical and social sciences, Applied General Systems Research (Internat. Conf., State Univ. New York, Binghamton, NY, 1977) (G. J. Klir, ed.), NATO Conf., Ser.: Systems Sci., vol. 5, Plenum, New York, 1978, pp. 575-587.

[6] Systems of differential inequalities and stochastic differential equations. IV, Journal of Mathematical Physics 19 (1978), no. 8, 1733-1741.

[7] _ Time lag versus stability, IEEE Transactions on Automatic Control 23 (1978), no. 1, $84-85$.

[8] Competitive processes and comparison differential systems. II, Journal of Mathematical and Physical Sciences 15 (1981), no. 5, 435-454.

[9] __ Stochastic stability analysis of model ecosystems with time-delay, Differential Equations and Applications in Ecology, Epidemics, and Population Problems (Claremont, Calif, 1981) (S. N. Busenberg and K. L. Cooke, eds.), Academic Press, New York, 1981, pp. 215-228.

[10] Stabilizing and oscillating hereditary and random structural perturbations effects on multispecies processes, Proceedings of Conference on Nonlinear Systems: Modelling, Simulation and Applications (S. B. Agase, ed.), The Publication of Science College, Nanded, 2003, pp. 1-20.

[11] L Large-scale stochastic hereditary systems under Markovian structural perturbations I: variational comparison theorem, to appear in Journal of Applied Mathematics and Stochastic Analysis. 
[12] _ Qualitative Analysis of Competitive-Cooperative Processes in Biological Physical and Social Sciences, (Monograph in preparation).

[13] G. S. Ladde and V. Lakshmikantham, Competitive-cooperative processes and stability of diffusion systems, Applied Stochastic Processes (Proc. Conf., Univ. Georgia, Athens, Ga, 1978) (G. Adomian, ed.), Academic Press, New York, 1980, pp. 83-108.

[14] _ Random Differential Inequalities, Mathematics in Science and Engineering, vol. 150, Academic Press, New York; Harcourt Brace Jovanovich, London, 1980.

[15] G. S. Ladde and B. A. Lawrence, Stability and convergence of stochastic approximation procedures under Markovian structural perturbations, Dynamic Systems and Applications 10 (2001), no. 2, $145-175$.

[16] _ Stability and convergence of large-scale stochastic approximation procedures under Markovian structural perturbations, Differential Equations and Dynamical Systems (D. Bahuguna, ed.), Narosa, New Delhi, 2004, pp. 25-48.

[17] G. S. Ladde and M. Sambandham, Stochastic Versus Deterministic Systems of Differential Equations, Monographs and Textbooks in Pure and Applied Mathematics, vol. 260, Marcel Dekker, New York, 2004.

[18] G. S. Ladde and D. D. Siljak, Multiplex control systems: stochastic stability and dynamic reliability, International Journal of Control 38 (1983), 515-524.

[19] V. Lakshmikantham and S. Leela, Differential and Integral Inequalities: Theory and Applications. Vol. I: Ordinary Differential Equations, Mathematics in Science and Engineering, vol. 55, Academic Press, New York, 1969.

[20] _ Differential and Integral Inequalities: Theory and Applications. Vol. II: Functional, Partial, Abstract, and Complex Differential Equations, Mathematics in Science and Engineering, vol. 55, Academic Press, New York, 1969.

[21] M. B. Nevel'son and R. Z. Has'minskiü, Stochastic Approximation and Recursive Estimation, Translations of Mathematical Monographs, vol. 47, American Mathematical Society, Rhode Island, 1973.

[22] D. D. Šiljak, Large-scale Dynamic Systems. Stability and Structure, North-Holland Series in System Science and Engineering, vol. 3, North-Holland, New York, 1978.

[23] M. T. Wasan, Stochastic Approximation, Cambridge Tracts in Mathematics and Mathematical Physics, no. 58, Cambridge University Press, London, 1969.

G. S. Ladde: Department of Mathematics, the University of Texas at Arlington, Arlington, TX 76019, USA

E-mail address: ladde@uta.edu 


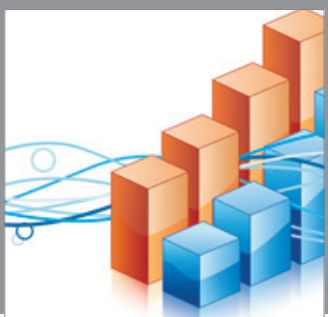

Advances in

Operations Research

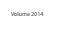

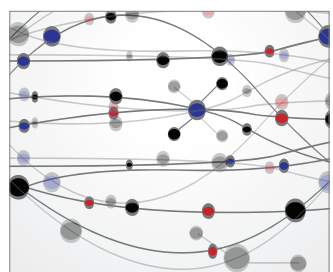

\section{The Scientific} World Journal
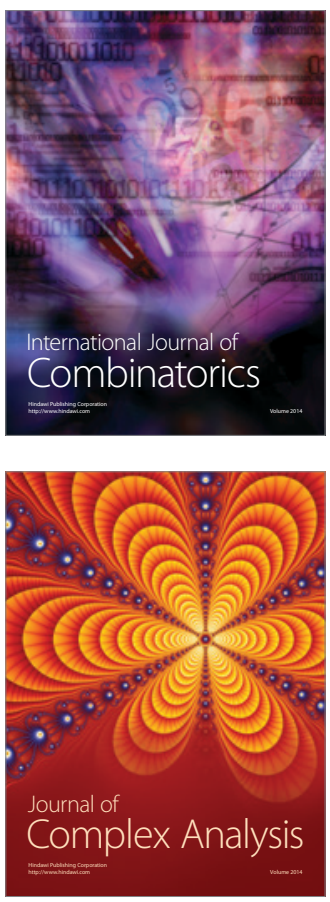

International Journal of

Mathematics and

Mathematical

Sciences
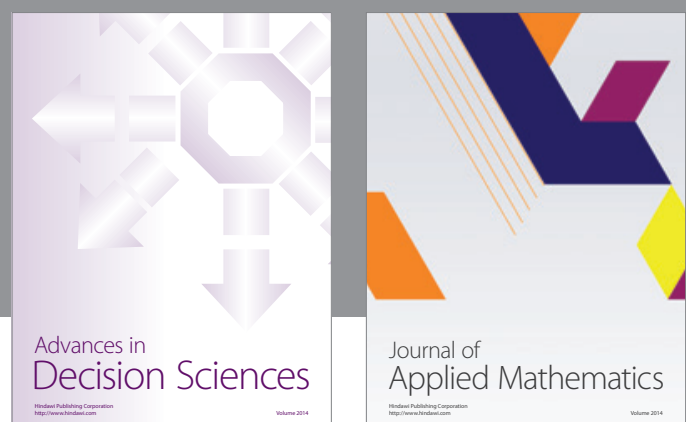

Journal of

Applied Mathematics
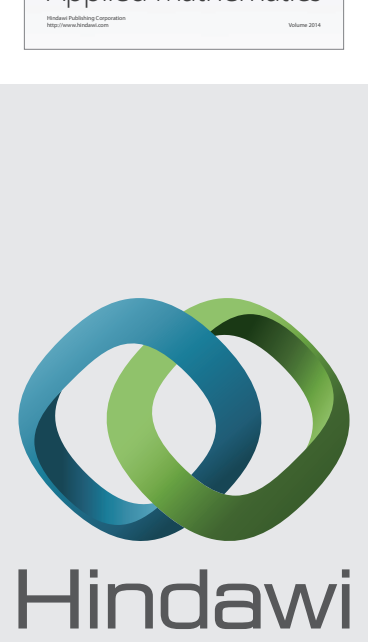

Submit your manuscripts at http://www.hindawi.com
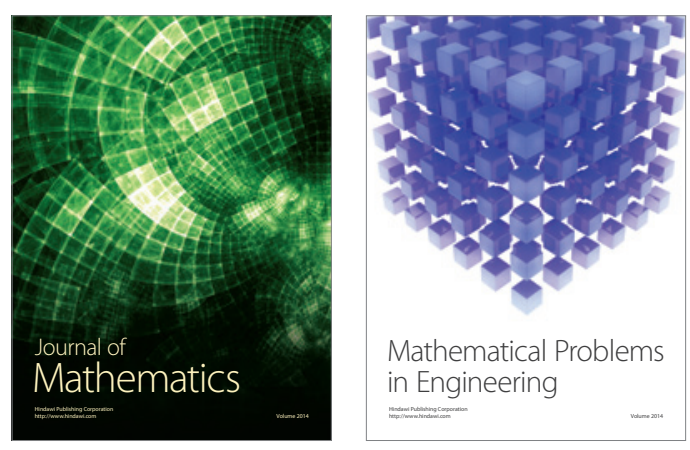

Mathematical Problems in Engineering
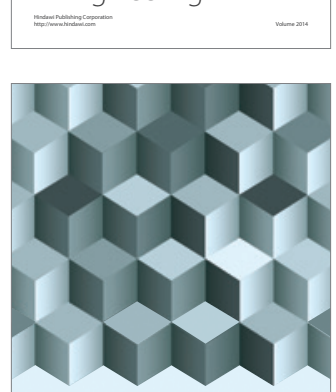

Journal of

Function Spaces
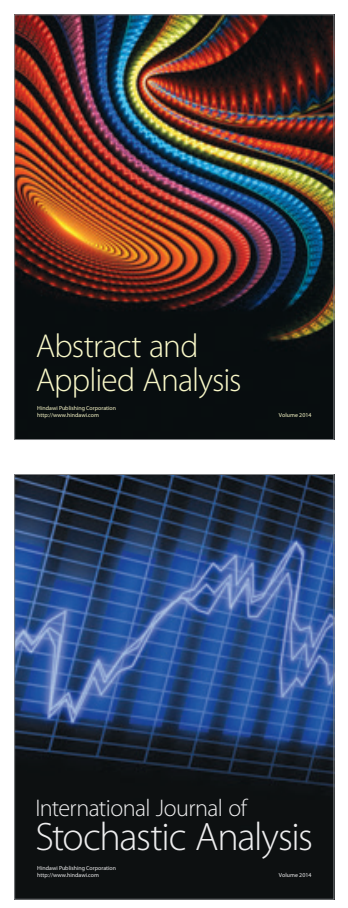

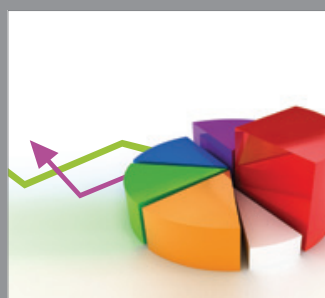

ournal of

Probability and Statistics

Promensencen
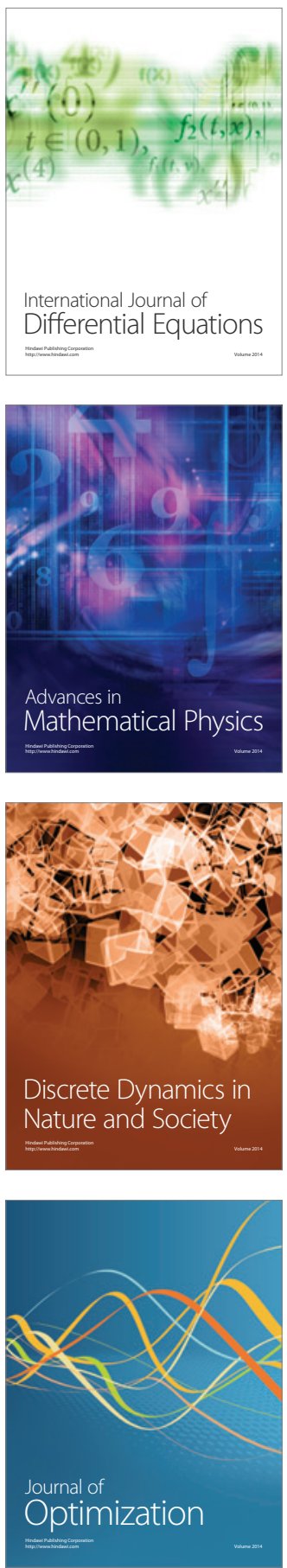